\title{
ESTIMATES OF THE INNER RADIUS OF UNIVALENCY OF DOMAINS BOUNDED BY CONIC SECTIONS
}

\author{
MATTI LEHTINEN
}

\section{Introduction}

Let $A$ be a simply connected domain in the extended plane, conformally equivalent to the unit disc. Denote by $M(A)$ the set of locally injective meromorphic functions in $A$ and by $\varrho_{A}$ the density of the Poincaré metric in $A$, so normalized that

$$
\varrho_{A}(h(z))\left|h^{\prime}(z)\right|=(2 \operatorname{Im} z)^{-1}
$$

if $h$ is a conformal map of the upper half-plane $H$ onto $A$. The set $Q(A)$ of the Schwarzian derivatives $S_{f}$ of $f \in M(A)$ is a Banach space with the norm

$$
\left\|S_{f}\right\|_{A}=\sup \left\{\left|S_{f}(z)\right| \varrho_{A}(z)^{-2} \mid z \in A\right\}
$$

The size of $\left\|S_{f}\right\|_{A}$ is connected to the global injectiveness of $f$. If $\left\|S_{f}\right\|_{A}=0$, $f$ is a Möbius transformation and hence univalent. If $A$ is Möbius equivalent to a disc, $\left\|S_{f}\right\|_{A} \leqq 2$ implies that $f$ is univalent and $\left\|S_{f}\right\|_{A}>6$ implies that $f$ is not univalent [8, 4]. Results due to Ahlfors [1] and Gehring [2] show that if $A$ is a quasidisc, then there exists a positive number $b$ such that $\left\|S_{f}\right\|_{A} \leqq b$ implies that $f$ is univalent and has a quasiconformal extension to the plane, and that this property characterizes quasidiscs.

Motivated by these results, Lehto [6] defined the inner radius of univalency $\sigma_{I}(A)$ of $A$ as the supremum (or maximum) of numbers $b$ such that every $f \in M(A)$ satisfying $\left\|S_{f}\right\|_{A} \leqq b$ is univalent. If $A$ is a quasidisc and $T(A) \subset Q(A)$ is the universal Teichmüller space of $A$, i.e., the set of $S_{f}$ such that $f$ has a quasiconformal extension to the plane, then $\sigma_{I}(A)$ is the radius of the largest ball centered at the origin of $Q(A)$ and contained in $T(A)$. Equivalently, $\sigma_{I}(A)$ is the distance of $S_{h}$ from the boundary of $T(H)$. Relatively little is known about the actual value of $\sigma_{I}(A)$ for a given domain $A$. The classical results of Nehari [8] and Hille [3] imply that $\sigma_{I}(A)=2$ for any domain $A$ Möbius equivalent to a disc, and Lehto [7] and the author [5] have observed that $\sigma_{I}(A)<2$ for all other simply connected domains. Denote by $A_{k}$ 
the angular domain $\{z|| \arg z \mid<k \pi / 2\}$. If $0<k \leqq 1$, one easily computes $\sigma_{I}\left(A_{k}\right)=$ $=2 k^{2}$. As pointed out by Lehto [7], the method employed by Ahlfors in proving that a quasidisc has a positive radius of univalency can be used to obtain explicit lower estimates for $\sigma_{I}(A)$, once a differentiable quasiconformal reflection of $A$, i.e., a sensereversing quasiconformal $\lambda: A \rightarrow A^{*}, A^{*}=\bar{C} \backslash \bar{A}$, fixing the boundary points of $A$, is known. More precisely,

$$
\sigma_{I}(A) \geqq 2 \inf _{z \in A} \frac{\left|\lambda_{\bar{z}}(z)\right|-\left|\lambda_{z}(z)\right|}{|\lambda(z)-z|^{2} \varrho_{A}(z)^{2}} .
$$

For an obtuse angle $A_{k}, \quad 1<k<2$, the natural reflection

yields

$$
\lambda(z)=-z(\bar{z} / z)^{1 / k}
$$

$$
\sigma_{I}\left(A_{k}\right) \geqq 4 k-2 k^{2}
$$

[7], where in fact equality holds [5]. Taking

$$
E_{r}=\left\{z \mid(r \operatorname{Re} z)^{2}+(\operatorname{Im} z)^{2}>1\right\}, 0<r \leqq 1,
$$

to be the outside of an ellipse with half-axes $1 / r, 1$, (1) applied to the reflection

with

$$
\lambda(z)=((1-r) / w+(1+r) / \bar{w}) /(2 r)
$$

gives

$$
z=((1+r) w+(1-r) / w) /(2 r)
$$

$$
\sigma_{I}\left(E_{r}\right) \geqq 8 r^{2} /(1+r)^{2}
$$

[7], which is asymptotically sharp for $r \rightarrow 0$ and $r \rightarrow 1$. (The last assertion is obvious, and if $\lim _{r \rightarrow 0} \sigma_{I}\left(E_{r}\right)=0$ were not true, there would exist a sequence $\left(r_{n}\right), r_{n} \rightarrow 0$, such that each $E_{r_{n}}$ would be a $K$-quasidisc with a fixed $K$ [2].)

\section{Domains bounded by a branch of a hyperbola}

There are simple cases in which a straightforward application of (1) produces no results. Consider, for example, the domain

$$
G=\left\{z \mid \operatorname{Re} z<0 \quad \text { or } \quad(\operatorname{Re} z)^{2}-(\operatorname{Im} z)^{2}<1\right\}
$$

bounded by a rectangular hyperbola. A natural 3-quasiconformal reflection $\lambda: G \rightarrow G^{*}$, continuously differentiable outside the asymptote rays of $\partial G$, is given 
by the formulas

$$
\lambda(z)=\left(2-\bar{z}^{2}\right)^{1 / 2}
$$

for those $z$ which lie in the domain bounded by $\partial G$ and the asymptotes of $\partial G$, and

$$
\lambda(z)=\left(2-e^{i \pi / 3} \bar{z}^{4 / 3} z^{2 / 3}\right)^{1 / 2}
$$

for $\pi / 4<\arg z<7 \pi / 4$. For this $\lambda$, however, the right-hand side of (1) equals zero.

For $c>0$, set

$$
G_{c}=\left\{z \mid \operatorname{Re} z<0 \quad \text { or }(c \operatorname{Re} z)^{2}-(\operatorname{Im} z)^{2}<1\right\} .
$$

Information on $\sigma_{I}\left(A_{k}\right)$ can be used to compute $\sigma_{I}\left(G_{c}\right)$ utilizing an idea introduced in $[5]$ :

Theorem 1. For every positive $c$,

$$
\sigma_{I}\left(G_{c}\right)=(8 / \pi) \arctan c-\left(8 / \pi^{2}\right)(\arctan c)^{2} .
$$

Proof. Let $k$ be an arbitrary number satisfying 2 arc $\tan c<k \pi<\pi$. Draw a circular arc in $G_{c}$, with both endpoints on the real axis and meeting the real axis at angle $k \pi / 2$, tangent to $\partial G_{c}$ at a point $z_{0}$. The infinite domain $T_{k}$ bounded by this arc and its mirror image in the real axis is Möbius equivalent to $A_{2-k}$, and $G_{c} \subset T_{k}$. By [5], a conformal map $f: T_{k} \rightarrow A_{2-k} \cap\left\{z \mid 1-z \in A_{2-k}\right\}$ satisfying $f\left(z_{0}\right)=f\left(\bar{z}_{0}\right)=\infty$ and $\left\|S_{f}\right\|_{T_{k}}=4(2-k)-2(2-k)^{2}=4 k-2 k^{2}$ exists. Since $\varrho_{G_{c}}(z)>\varrho_{T_{k}}(z)$ for all $z \in G_{c},\left\|S_{f}\right\|_{G_{c}} \leqq 4 k-2 k^{2}$. Also, $f\left(G_{c}\right)$ is not a Jordan domain. Consequently,

$$
\sigma_{I}\left(G_{c}\right) \leqq(8 / \pi) \arctan c-\left(8 / \pi^{2}\right)(\arctan c)^{2} .
$$

To prove the opposite inequality, observe that for every $\varepsilon>0$, there exists an $f \in M\left(G_{c}\right)$ such that $\left\|S_{f}\right\|_{G_{c}}<\sigma_{I}\left(G_{c}\right)+\varepsilon$ and $f$ is not univalent. Assume $f\left(z_{1}\right)=f\left(z_{2}\right)$, $z_{1} \neq z_{2}$. Then either $z_{1}$ and $z_{2}$ are in the closure of a disc or half-plane $U \subset G_{c}$ or in the closure of an angular domain $U \subset G_{c}$ Möbius equivalent to $A_{2-k}$ for some $k$, 2 arc $\tan c<k \pi<\pi$. ( $U$ is bounded, for example, by tangents to $\partial G_{c}$ through $z_{1}$ and $z_{2}$.) Since $f$ is not univalent in the closure of $U,\left\|S_{f}\right\|_{U} \geqq \sigma_{I}(U) \geqq 4 k-2 k^{2}$. Again taking into account the monotonicity of the Poincaré metric with respect to domain, one gets $\left\|S_{f}\right\|_{G_{c}} \geqq\left\|S_{f}\right\|_{U}$, and the inequality opposite to (4) follows.

We next consider the domain $G_{c}^{*}$, complementary to $G_{c}$. Reasoning as above, we obtain

Theorem 2. For every positive $c$,

$$
\sigma_{I}\left(G_{c}^{*}\right)=\left(8 / \pi^{2}\right)(\arctan c)^{2} .
$$

Proof. Let $k \pi=2 \arctan c$. Then $G_{c} \subset A_{k}$. The function $g, g(z)=\log z$, maps $A_{k}$ onto an infinite horizontal strip of width $k \pi$, and $g\left(\partial G_{c}^{*}\right)$ is not a quasicircle. Since $\left\|S_{g}\right\|_{A_{k}}=2 k^{2}, \sigma_{I}\left(G_{c}\right) \leqq 2 k^{2}$. On the other hand, assume $f \in M\left(G_{c}\right)$ with $\left\|S_{f}\right\|_{G_{c}}<\sigma_{I}\left(G_{c}\right)+\varepsilon$ satisfies $f\left(z_{1}\right)=f\left(z_{2}\right)$. Then, considering the rays parallel to the 
asymptotes of $\partial G_{c}^{*}$ which join $z_{1}$ and $z_{2}$ to $\infty$ in $G_{c}^{*}$ and the segment joining $z_{1}$ to $z_{2}$, we observe that $z_{1}$ and $z_{2}$ are on the boundary of a finite domain $U$ bounded by a segment and a circular arc meeting at an angle at least $k \pi$. Since $U$ is Möbius equivalent to some $A_{k^{\prime}}, k^{\prime} \geqq k$, we obtain $\sigma_{I}\left(G_{c}\right) \geqq 2 k^{2}$ as in the proof of Theorem 1 .

\section{The exterior of an ellipse}

The method employed above for domains bounded by a branch of a hyperbola does not yield the exact value of $\sigma_{I}\left(E_{r}\right)$. However, (3) can be improved considerably, and an upper bound can be established, too.

Theorem 3. For every $r, 0<r \leqq 1$,

$$
\begin{gathered}
(16 / \pi) \arctan q-\left(32 / \pi^{2}\right)(\arctan q)^{2} \leqq \sigma_{I}\left(E_{r}\right) \\
\leqq(16 / \pi) \arctan r-\left(32 / \pi^{2}\right)(\arctan r)^{2},
\end{gathered}
$$

where $q=r /\left(2-r^{2}\right)^{1 / 2}$.

Proof. Denote by $T_{r}$ the infinite domain bounded by two circular arcs through $1 / r, i,-1 / r$ and $1 / r,-i,-1 / r$, respectively. Then $E_{r} \subset T_{r},\{i,-i\} \subset \partial T_{r} \cap \partial E_{r}$, and $T_{r}$ is, by elementary geometry, Möbius equivalent to $A_{k}, k=2-(4 / \pi) \arctan r$. By [5], a conformal map of $T_{r}$ onto a non-quasidisc exists such that $f(i)=f(-i)$ and $\left\|S_{f}\right\|_{T_{r}}=4 k-2 k^{2}$. As in Theorem 1 , we deduce $\sigma_{I}\left(E_{r}\right) \leqq 4 k-2 k^{2}$, or the righthand side of (5).

To prove the left-hand side of (5), assume $f \in M\left(E_{r}\right),\left\|S_{f}\right\|_{E_{r}}<\sigma_{I}\left(E_{r}\right)+\varepsilon$, and $f\left(z_{1}\right)=f\left(z_{2}\right), z_{1} \neq z_{2}$. Consider discs $D_{j}, j=1,2$, such that $E_{r}^{*} \subset D_{j}, z_{j} \in \partial D_{j}$ and $\partial D_{j}$ touches $\partial E_{r}$ either at two points symmetric with respect to the imaginary axis or one of the points $i,-i$. If $z_{1} \notin D_{2}$ or $z_{2} \notin D_{1}$, both $z_{1}$ and $z_{2}$ belong to the closure of a domain $U \subset E_{r}$ such that $\sigma_{I}(U)=2$. Otherwise, set $U=\left(D_{1} \cap D_{2}\right)^{*}$. Then, by an elementary geometric argument, $U$ is Möbius equivalent to an $A_{k}, 1<k<$ $2-(4 / \pi)$ arc $\tan q$. In each case, $f$ maps $U$ onto a domain not bounded by a Jordan curve or $f$ is not injective. Hence $\left\|S_{f}\right\|_{E_{r}} \geqq\left\|S_{f}\right\|_{U} \geqq 4 k-2 k^{2}$, and the left-hand side of (5) follows.

Acknowledgement. The author wishes to thank David Calvis for his useful comments. 


\section{References}

[1] AHLfors, L. V.: Quasiconformal reflections. - Acta Math. 109, 1963, 291-301.

[2] Gehring, F. W.: Univalent functions and the Schwarzian derivative. - Comment. Math. Helv. 52, $1977,561-572$.

[3] Hille, E.: Remarks on a paper by Zeev Nehari. - Bull. Amer. Math. Soc. 55, 1949, 552-553.

[4] KraUs, W.: Über den Zusammenhang einiger Charakteristiken eines einfach zusammenhängenden Bereichs mit der Kreisabbildung. - Mitt. Math. Sem. Univ. Giessen 21, 1932, 1-26.

[5] Lehtinen, M.: On the inner radius of univalency for noncircular domains. - Ann. Acad. Sci. Fenn. Ser. A I Math. 5, 1980, 45-47.

[6] LeHTo, O.: Domain cons ants associated with Schwarzian derivative. - Comment. Math. Helv. 52, 1977, 603-510.

[7] LeHTo, O.: Remarks on Nehari's theorem about the Schwarzian derivative and schlicht functions. - J. Analyse Math. 36, 1979, 184-190.

[8] NehARI, Z.: The Schwarzian derivative and schlicht functions. - Bull. Amer. Math. Soc. 55, 1949, 545-551.

University of Helsinki

Department of Mathematics

SF-00100 Helsinki

Finland

Received 23 May 1983 